\title{
Backflow in the relaxation of a hybrid aligned nematic cell
}

\author{
S. A. Jewell a) and J. R. Sambles \\ Thin Film Photonics Group, School of Physics, University of Exeter, Stocker Road, Exeter, \\ EX4 4QL UK England
}

(Received 27 January 2003; accepted 17 March 2003)

\begin{abstract}
The optical convergent-beam technique has been used to measure the changing director profile in a $4.6 \mu \mathrm{m}$ ZLI-2293 filled hybrid aligned nematic cell when a $7 \mathrm{~V}_{\mathrm{rms}}$ ac voltage was removed. The relaxation process has been recorded in $0.3 \mathrm{~ms}$ time steps allowing the detailed director backflow occurring in the initial $9 \mathrm{~ms}$ of the reorientation process to be quantified. The measured tilt profiles over the $60 \mathrm{~ms}$ total relaxation period were compared to model tilt profiles produced using the Leslie-Eriksen-Parodi theory, and excellent agreement was found. Further analysis shows that the backflow is dominated by the viscosity coefficient $\eta_{1}$ and the overall relaxation is governed by the coefficient $\gamma_{1}$. (C) 2003 American Institute of Physics. [DOI: 10.1063/1.1573336]
\end{abstract}

The hybrid aligned nematic (HAN) geometry, comprised of homogeneous alignment on one surface and homeotropic on the other, is currently of great commercial interest as it forms one of the two stable states of the low-power zenithal bistable display. The cell structure leads to the dynamic response of the director to changes in the applied voltage being considerably faster than that of a comparable twisted nematic cell. Thus characterization of this reorientation process in detail is of substantial interest.

The hydrodynamics associated with a liquid crystal is far more complex than that of an isotropic fluid due to a coupling between the director and fluid flow during any change in the alignment of the molecules. This is described by the Leslie-Eriksen-Parodi (LEP) theory, which involves the use of five independent viscosity coefficients associated with flow in various directions relative to the director. ${ }^{1-4}$

The LEP equations for the relaxation from an applied voltage for a nontwisted HAN cell were solved numerically and the results suggest that "backflow" occurs in the dynamic process. For a liquid crystal of positive dielectric anisotropy, the director in a HAN cell with an ac voltage applied is predominantly homeotropically aligned. On removal of the voltage, the director close to the homeotropic surface initially rotates past the $0 \mathrm{~V}$ equilibrium tilt angle associated with that region of the cell before relaxing back to its $0 \mathrm{~V}$ state. This backflow occurs over the initial few milliseconds of the reorientation process, and is driven by a coupling between shear and rotational fluid motion in adjacent regions of the cell.

To allow this backflow to be observed experimentally, a technique is required that can measure subtle changes in the director profile on a submillisecond time scale. Optical waveguide characterization techniques have been used for several years to measure the static director profile in liquid crystal cells. ${ }^{5-8}$ More recently, this optical waveguide technique has been modified by using an incident beam that is expanded and then focused down to a spot, allowing optical modes to be simultaneously excited in the cell over a wide angle range. Optical intensity versus angle-of-incidence data are then collected for reflected and transmitted light by using a charge coupled device array. ${ }^{9-11}$ This is known as the convergent-beam technique and allows the liquid crystal dynamics to be recorded on a $0.3 \mathrm{~ms}$ time scale, which is substantially lower than the total response time of a cell, whilst maintaining the high-quality director-profile information associated with static optical characterization techniques.

A 4.6- $\mu$ m-thick HAN cell was constructed and filled with the nematic liquid crystal compound ZLI-2293 (Merck). A $15 \mathrm{~nm}$ layer of silicon monoxide obliquely evaporated at a $60^{\circ}$ angle of incidence provides homogeneous alignment on one indium tin oxide surface, and a monolayer of octadecyltrimethoxysilane on the other provides homeotropic alignment (Fig. 1). The cell was mounted between two low-index hemispheres at a $60^{\circ}$ azimuthal angle, and centred in a convergent-beam system, as shown in Fig. 2.

A $10 \mathrm{kHz}$ ac $7 \mathrm{~V} \mathrm{rms}$ voltage was applied across the cell and optical intensity versus angle of incidence data was collected as the cell relaxed to the $0 \mathrm{~V}$ state, a process taking around $60 \mathrm{~ms}$. The room temperature $\left(23^{\circ} \mathrm{C}\right)$ datasets were collected for transmission and reflection for polarization conserving signals ( $p$-to- $p$ (transverse magnetic) and $s$-to- $s$ (transverse electric) and polarization converting signals ( $p$-to-s and $s$-to- $p$ ) as shown in Fig. 3.

By splitting the data into time steps, a complete set of optical data (four transmitted and four reflected datasets) was obtained for the director profile in $0.3 \mathrm{~ms}$ increments following the removal of the voltage. The director profile at each point in time was then treated as a static profile and the data was fitted to using a multilayer optics least-squares fitting

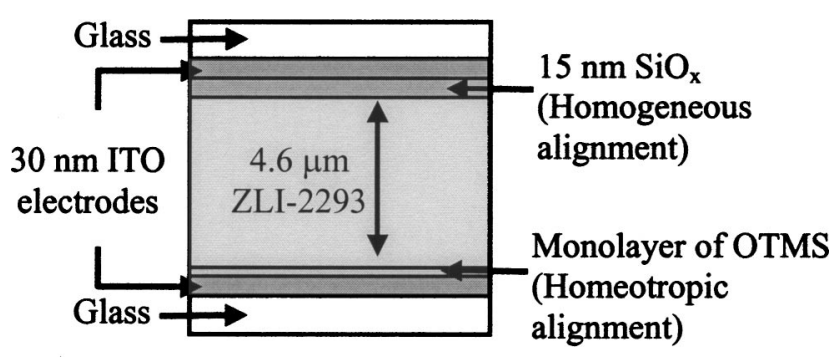

FIG. 1. A schematic of the structure of the HAN cell used.

${ }^{\text {a)} E l e c t r o n i c ~ m a i l: ~ s . a . j e w e l l @ e x e t e r . a c . u k ~}$ 


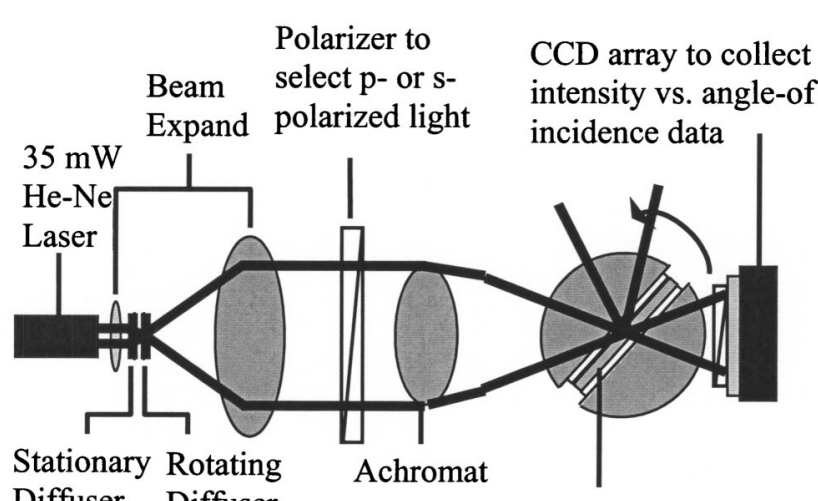

Diffuser Diffuser
Cell index matched between two low-index $(n=1.52)$ hemispheres

FIG. 2. Schematic diagram of the layout of the optical components used in the convergent beam technique.

routine, based on a Berreman $4 \times 4$ matrix approach. ${ }^{12}$ The optical permittivity, absorption, and thickness of each optical layer were used as fitting parameters, along with the tilt profile of the liquid crystal layer.

Fitting to the static tilt profiles at $t=0(7 \mathrm{~V}$ applied $)$ and $t=70 \mathrm{~ms}(0 \mathrm{~V})$ produced tilt profiles showing excellent agreement with model profiles produced by a free-energy minimization static tilt profile model. The results of fitting to a selection of lines of dynamic data are shown in Fig. 4. During the fitting procedure the director profile, cell thickness and liquid crystal optical permittivities were allowed to vary.

A comparison between the measured tilt profiles and modeled tilt profiles produced using viscosity coefficients of $\eta_{1}=0.160 \pm 0.005 \mathrm{mPas}, \quad \eta_{2}=0.010 \pm 0.005 \mathrm{mPas}$, $\eta_{12}=-0.10 \pm 0.05 \mathrm{mPas}$, and $\gamma_{1}=0.155 \pm 0.005 \mathrm{mPas}$ are shown in Fig. 5. These values were obtained by comparing the measured profiles to model profiles generated using a program based on the LEP theory. During the switch-off process, backflow is clearly seen in the first $9 \mathrm{~ms}$ for the region
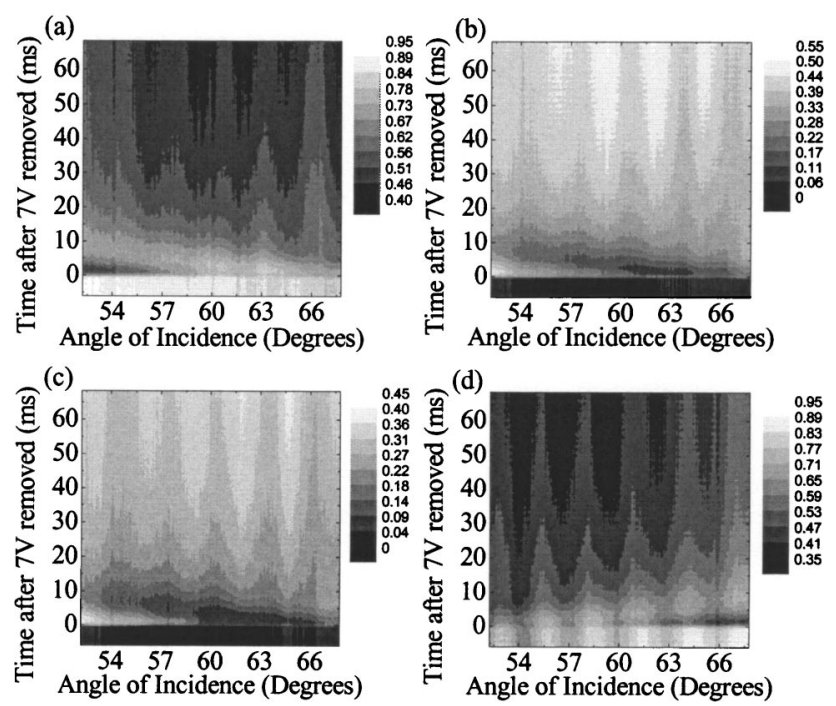

FIG. 3. Gray-scale plots showing the variation in optical intensity vs angleof-incidence data with time after a $7 \mathrm{~V}$ ac rms voltage was removed from the HAN cell for the transmitted data sets (a) $T_{\mathrm{pp}}$, (b) $T_{\mathrm{ps}}$, (c) $T_{\mathrm{sp}}$, and (d) $T_{\mathrm{ss}}$.
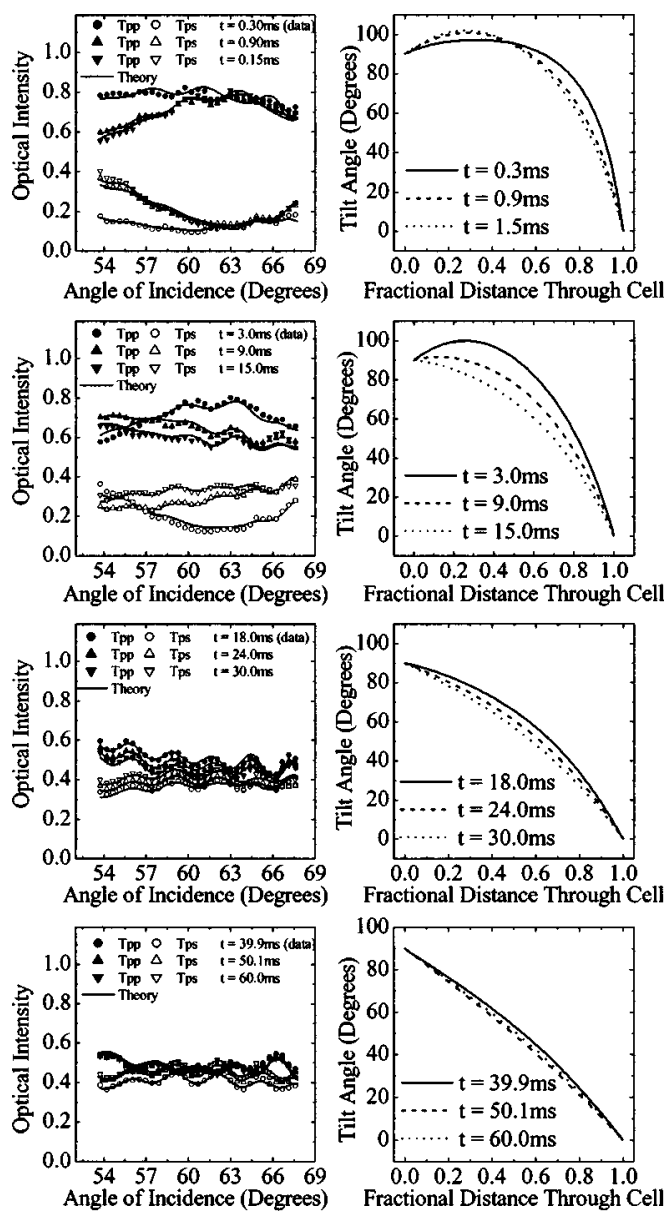

FIG. 4. Comparison between measured optical-intensity vs angle-ofincidence data (symbols) and theoretical data (lines) produced using a multilayer optics model for a selection of times after the removal of the voltage.

adjacent to the homeotropically aligned surface. Excellent agreement is seen between the measured and modeled tilt profiles of the HAN cell. Further modeling showed that the two most influential viscosity coefficients are $\eta_{1}$, which govern the amount of backflow occurring during the initial reorientation, and $\gamma_{1}$ which determines the overall relaxation time of the cell.

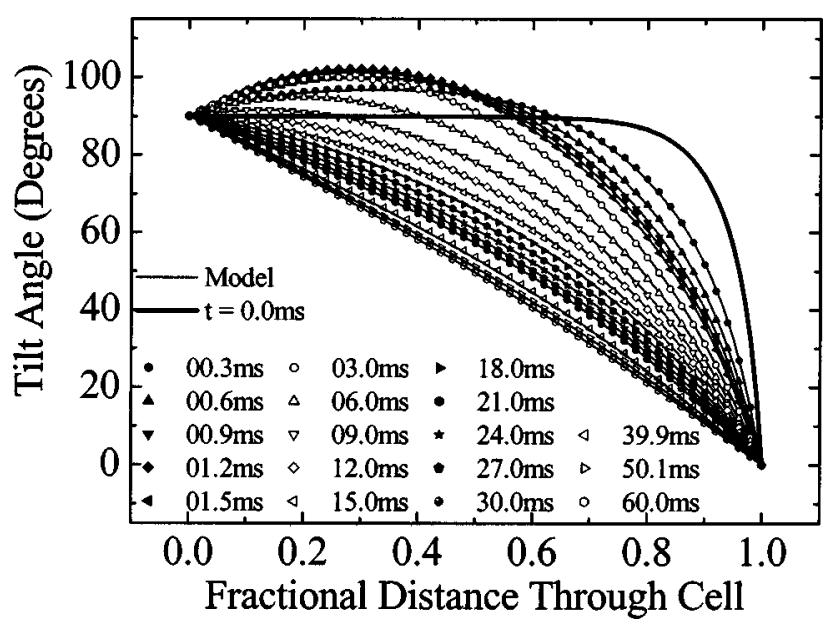

FIG. 5. Comparison between measured tilt profiles (symbols) and model profiles (lines) generated using the LEP nematodynamics model for various times after the removal of the applied voltage. Backflow can clearly be seen occurring in the initial $9 \mathrm{~ms}$ after the removal of the voltage.

AIP license or copyright; see http://apl.aip.org/apl/copyright.jsp 
In conclusion, the detailed director profile in a HAN cell relaxing from an applied voltage in submillisecond time steps has been obtained. The total relaxation time for the 4.6 $\mu \mathrm{m}$ ZLI-2293 cell from an applied ac voltage of $7 \mathrm{~V}$ is of order $60 \mathrm{~ms}$ and, as predicted by the LEP theory, backflow occurs in the first $9 \mathrm{~ms}$ after the voltage is removed.

The profiles measured show excellent agreement with model profiles produced using a nematodynamics modeling program, and the viscosity coefficients used are in agreement with previously published values. Further analysis has shown that the backflow is governed by the shear viscosity coefficient $\eta_{1}$ and the overall relaxation time is determined by the rotational viscosity coefficient $\gamma_{1}$.
The authors wish to thank EPSRC and Hewlett-Packard Laboratories, Bristol for the financial support of this work.

${ }^{1}$ J. L. Eriksen, Trans. Soc. Rheol. 5, 23 (1961).

${ }^{2}$ J. L. Eriksen, Arch. Ration. Mech. Anal. 9, 371 (1962).

${ }^{3}$ F. M. Leslie, Q. J. Mech. Appl. Matn. 19, 357 (1966).

${ }^{4}$ O. Parodi, J. Phys. (Paris) 31, 581 (1970).

${ }^{5}$ F. Z. Yang and J. R. Sambles, J. Opt. Soc. Am. B 16, 3 (1999).

${ }^{6}$ B. T. Hallam, F. Yang, and J. R. Sambles, Liq. Cryst. 26, 5 (1999).

${ }^{7}$ F. Yang, L. Ruan, and J. R. Sambles, J. Appl. Phys. 88, 11 (2000).

${ }^{8}$ S. A. Jewell and J. R. Sambles, J. Appl. Phys. 92, 1 (2002).

${ }^{9}$ N. J. Smith and J. R. Sambles, Appl. Phys. Lett. 77, 2632 (2001).

${ }^{10}$ N. J. Smith, M. D. Tillin, and J. R. Sambles, Phys. Rev. Lett. 88, 088301 (2002).

${ }^{11}$ L. Ruan and J. R. Sambles, J. Appl. Phys. 92, 4857 (2002).

${ }^{12}$ D. Y. K. Ko and J. R. Sambles, J. Opt. Soc. Am. A 5, 1863 (1988). 